Bio - grafía. Escritos sobre la Biología y su Enseñanza. ISSN 2027-1034

Edición Extraordinaria. p.p. 918 - 925

Memorias del IX Encuentro Nacional de Experiencias en Enseñanza de la Biología y la Educación Ambiental. IV Congreso Nacional de Investigación en Enseñanza de la Biología.

\title{
LA ENSEÑANZA DEL SISTEMA DIGESTIVO Y NUTRICIÓN A TRAVÉS DEL ENFOQUE DE INVESTIGACIÓN DIRIGIDA
}

\section{TEACHING THE DIGESTIVE SYSTEM AND NUTRTION THROUGH THE TARGETED RESEARCH APPROACH}

\author{
Eva Yarina Rueda García \\ Raul David Piraquive Bermúdez
}

\section{RESUMEN:}

Esta ponencia está centrada en evidenciar, cómo el enfoque de investigación dirigida aporta al aprendizaje del sistema digestivo y nutrición en los estudiantes de grado 701 del colegio IED Toberin, ubicado en la localidad de Usaquén, Bogotá, Colombia. En este trabajo se aplica una metodología cualitativa; donde se realiza la implementación de una unidad didáctica, contenida con unas actividades específicas sobre aparato digestivo y nutrición, a las cuales se les realiza un seguimiento mediante técnicas de recolección de datos como:observación participante, análisis documental, entrevistas y encuestas. Finalmente, se evidencia como se formó una memoria final en la cual los alumnos son capaces, no solo de examinar los resultados obtenidos frente a un problema planteado, sino también, de analizar y elegir sus propias estrategias de resolución.

PALABRAS CLAVE: Aprendizajes conceptuales, actitudinales y procedimentales, enfoque pedagógico, ideas previas, sistema digestivo, nutrición.

\section{ABSTRACT:}

This paper is focused on evidencing how the directed research approach contributes to the digestive system and nutrition learning in the 701 grade students of IED Toberin, located in Usaquén's, Bogotá, Colombia. In this work, a qualitative methodology is applied; Where the implementation of a didactic unit is carried out, with specific activities on the digestive system and nutrition, which are followed up by techniques of data collection such as: participant observation, documentary analysis, interviews, and surveys. Finally, it is evident how a final memory was formed in which students are able not only to examine the results obtained in the face of a problem, but also to analyze and choose their own strategies for resolution

\section{KEY WORD:}

conceptual, attitudinal and procedural learning, pedagogical approach, previous ideas, digestive system, nutrition. 
Bio - grafía. Escritos sobre la Biología y su Enseñanza. ISSN 2027-1034

Edición Extraordinaria. p.p. 918 - 925

Memorias del IX Encuentro Nacional de Experiencias en Enseñanza de la Biología y la

Educación Ambiental. IV Congreso Nacional de Investigación en Enseñanza de la Biología.

\section{INTRODUCCIÓN}

Todos los seres humanos nacen con la capacidad de aprender, lo que les permite actuar en el entorno y reaccionar frente a éste. Sabemos que la ciencia es considerada un proceso de construcción de la realidad, la cual no se tiene que aprender memorísticamente, sino que se va construyendo poco a poco; es importante fomentar en los estudiantes un espíritu científico ya que la motivación y los estímulos favorecen el aprendizaje de las ciencias. (Pozo, 2004).

Desde la historicidad del concepto "sistema digestivo"encontramos que los primeros registros sobre el proceso digestivo se deben a Hipócrates de Cos (460-377 a.C.), quien explica que la digestión es una serie de acciones de naturaleza mecánica, llevada a cabo por la masticación del alimento y por un supuesto proceso de cocción en el estómago, donde se separaban los componentes aprovechables y no aprovechables para el organismo (Sánchez, 2013).

Ahora bien,el enfoque de investigación dirigida,busca lograr el cambio en la mente de los alumnos desde los ámbitos tanto conceptuales como metodológicos y actitudinales, para ello debe situárseles en un contexto similar al que vive un científico para lograr alc anzar los objetivos esperados, pero bajo la atenta dirección del profesor que, al igual que sucedería en el enfoque de enseñanza por descubrimiento, actuaría como "director de investigaciones. (Gil, 1993). Las actividades de evaluación en el modelo de investigación dirigida, se dan por medio de resolución de situaciones problema tanto el estudiante como el profesor, los cuales deben tener como característica situaciones abiertas que exijan búsqueda de nuevas respuestas, donde se integren los aspectos cualitativos y los cuantitativos. (Pozo, 2004). Referente a lo que se considera un aprendizaje conceptual encontramos que Pozo y Gómez 2004, Afirman:

bastaría con destacar que los hechos y los datos se aprenden de modo literal, consisten en una reproducción exacta, en la que el aprendiz no pone nada de su parte, salvo en el esfuerzo de repetirla, mientras que los conceptos se aprenden relacionándolos con los conocimientos previos que se poseen. (p.90).(Pozo y Gómez;2004).

En este trabajo los conceptos que se espera que el alumno aprenda son: macromoléculas, órganos del sistema digestivo, ruta de metabolismo y nutrición. Conrespecto a las actitudes están basadas en el valor del respeto para alcanzar la competencia. En tanto al aprendizaje procedimental (Morales, et al; 20138, Afirman:

El aprendizaje de procedimientos y procesos, está relacionado al "saber hacer", por tanto, es un paso posterior a la adquisición de datos y conceptos. El saber hacer, requiere por lo general realizar una secuencia de pasos, o secuencia de acciones para lo cual se requiere la adquisición de las habilidades y destrezas necesarias, los elementos que intervienen y cómo trabajarlos. (p.5).

Y por último en cuanto al aprendizaje actitudinal encontramos que Morales, et al; 2013, Afirman que: 


\title{
Bio - grafía. Escritos sobre la Biología y su Enseñanza. ISSN 2027-1034
}

Edición Extraordinaria. p.p. 918 - 925

\author{
Memorias del IX Encuentro Nacional de Experiencias en Enseñanza de la Biología y la \\ Educación Ambiental. IV Congreso Nacional de Investigación en Enseñanza de la \\ Biología.
}

Las actitudes y valores están en todo proceso de aprendizaje y suelen ser trabajadas de forma transversal. Una vez adquirido el aprendizaje de conceptos y procesos, permiten valorar la adecuada aplicación de habilidades y destrezas ante un determinado caso o problema, de esta manera se puede comprobar si los conocimientos adquiridos a nivel conceptual, procedimental y actitudinal, han sido suficientes para alcanzar la competencia o subcompetencia. (p.5).(Morales, et al; 2013).

En este trabajo, se fomenta la capacidad de formular hipótesis y resolverlas por medio de la investigación directa (prácticas de laboratorio), investigación teórica y audiovisual (análisis de documentos, videos e indagaciones propias), todo esto bajo el marco del respeto mutuo y propio, el trabajo en equipo, el interés por el aprendizaje y la actitud investigativa.

En el marco de la investigación de la enseñanza del sistema digestivo observamos cómo en la investigación realizada por Cubero: "Aprendizaje de la digestión en la enseñanza primaria", demuestra que la mayoría de las dificultades que tienen los estudiantes acerca del aprendizaje del sistema digestivo es debido a las experiencias cotidianas y medios de comunicación lo cual ha hecho pensar a los niños y niñas que el estomago es el órgano principal y dejen en un segundo plano a los demás. Otra parte importante es la relacionada con la expulsión de los desechos donde los estudiantes creen que la comida no sale nunca del cuerpo o que puede ser eliminada oralmente (vomitando). Además de la organización dentro del cuerpo, por ejemplo, cuando los niños realizan sus dibujos aparece una especie de bolsa (estomago) desconectada de otros elementos (órganos) del interior del cuerpo. (Cubero, 1998)

Banet, E. y Núñez, F. en 1992: como resultado de sus investigaciones plantean la siguiente propuesta de enseñanza, la cual tiene como propósito lograr que los alumnos aprendan significativamente los principales aspectos relacionados con la digestión de los alimentos, es decir, modificar sus ideas erróneas y ampliar sus esquemas conceptuales. Ubican su propuesta en tres categorías: 1) anatomía del aparato digestivo. 2) descomposición de las sustancias nutritivas contenidas en los alimentos. 3) absorción de los productos resultantes de la digestión, los cuales comprenden las dificultades más significativas de los alumnos. Tomando sus ideas y conocimiento como eje central para que el docente ejecute las actividades que le parezcan pertinentes teniendo en cuenta las necesidades de los alumnos las cuales pueden modificar el orden de aplicación y reestructurar las actividades.

\section{METODOLOGÍA}

La metodología de este trabajo al ser cualitativa orienta a renovar constantemente la praxis pedagógica.El docente investigador es transformador, partiendo de los "por qué" y los "para qué" que le permitan orientar sus fines, resultado de la observación e interpretación de las particularidades de la escuela como objeto social, permitiendo una observación participativa. Esta metodología se divide en tres fases para la 
Bio - grafía. Escritos sobre la Biología y su Enseñanza. ISSN 2027-1034

Edición Extraordinaria. p.p. 918 - 925

Memorias del IX Encuentro Nacional de Experiencias en Enseñanza de la Biología y la Educación Ambiental. IV Congreso Nacional de Investigación en Enseñanza de la Biología.

implementación de los instrumentos y la recolección de datos, la cuales son: Fase de caracterización, Fase de articulación e implementación y Fase recolección de los datos y análisis. Como fase de caracterización, la población se localiza en el colegio IED Toberin, ubicado en la localidad de Usaquén, Bogotá, Colombia, grado 701; se realiza una encuesta a los estudiantes donde se analizaron datos como: situación socio económica, preferencias académicas, artísticas y deportivas e ideas previas respecto al tema de sistema digestivo y nutrición.En la fase de articulación e implementación, luego de la caracterización de la población se elabora e implementa una unidad didáctica donde se presentan las siguientes actividades:

\begin{tabular}{|c|c|}
\hline NOMBRE DE LA ACTIVIDAD & $\begin{array}{c}\text { DESCRIPCION DE LAS ESTRATEGIAS } \\
\text { DE ENSEÑANZA }\end{array}$ \\
\hline $\begin{array}{c}\text { Macro moléculas la fuente de nuestra } \\
\text { energía, Siguiendo la ruta de nuestro } \\
\text { metabolismo }\end{array}$ & $\begin{array}{l}\text { Introducción al tema por medio de la } \\
\text { presentación de una situación problemática } \\
\text { (¿cuáles son las principales } \\
\text { macromoléculas en la digestión? y iqué } \\
\text { sucede si comemos alimentos } \\
\text { contaminados o con las manos sucias?), } \\
\text { promoviendo la generación de una } \\
\text { respuesta a la hipótesis con ayuda de un } \\
\text { video visto en clase y los conceptos y } \\
\text { apuntes obtenidos en la clase anterior. } \\
\text { Generando grupos de trabajo para que } \\
\text { permita un reforzamiento actitudinal y } \\
\text { procedimental. }\end{array}$ \\
\hline Me aproximo al laboratorio & $\begin{array}{l}\text { Formulación de una hipótesis acerca del } \\
\text { tipo de macromoléculas que encontramos } \\
\text { en alimentos de uso común, gracias a los } \\
\text { conocimientos adquiridos previamente lo } \\
\text { que permitirá el reforzamiento conceptual, } \\
\text { desarrollándose la primera parte de la "UVE } \\
\text { HEURISTICA". }\end{array}$ \\
\hline & \\
\hline
\end{tabular}


Bio - grafía. Escritos sobre la Biología y su Enseñanza. ISSN 2027-1034

Edición Extraordinaria. p.p. 918 - 925

Memorias del IX Encuentro Nacional de Experiencias en Enseñanza de la Biología y la Educación Ambiental. IV Congreso Nacional de Investigación en Enseñanza de la Biología.

\begin{tabular}{|c|l|}
\hline $\begin{array}{c}\text { Determinación de la presencia de } \\
\text { carbohidratos, lípidos y proteínas en } \\
\text { alimentos naturales de uso común }\end{array}$ & $\begin{array}{l}\text { Experimentación para comprobar la } \\
\text { hipótesis, por medio de la organización de } \\
\text { los datos y resultados obtenidos (todo esto } \\
\text { manteniendo los grupos de trabajo, } \\
\text { fortaleciendo el trabajo en equipo y la } \\
\text { colaboración conjunta para facilitar la } \\
\text { solución de problemas) finalización de la } \\
\text { "UVE HEURISTICA" }\end{array}$ \\
\hline La nutrición es salud & $\begin{array}{l}\text { Formular una hipótesis a partir de una } \\
\text { pregunta orientadora dada en clase lo cual } \\
\text { dará paso a la investigación, reforzamiento } \\
\text { finalización del concepto de sistema } \\
\text { digestivo y nutrición ya que se abarca todo } \\
\text { visto durante el desarrollo de las } \\
\text { loctividades. }\end{array}$ \\
&
\end{tabular}

Tabla 1. Actividades y estrategias de enseñanza, (Piraquive y Rueda, 2017)

En la medida en que se implementaban las actividades anteriormente mencionadas, se realizó la fase de análisis y recolección de datos por medio de las siguientes técnicas: Observación participativa, la cual se utiliza en todas las actividades ya que, al cumplir un papel de director de investigación, propiciamos un ambiente investigativo que nos permitió hacer un seguimiento constante a la evolución del conocimiento conceptual, actitudinal y procedimental de los estudiantes. Análisis documental, el cual nos proporciona una evidencia del avance conceptual de los estudiantes por medio de los documentos y tareas que estos realizaron, esta técnica se aplica durante el desarrollo de las actividades, al iniciar y al finalizarlas con el fin de poder evidenciar el progreso de los estudiantes descrito con sus propias palabras.

\section{RESULTADOS Y DISCUSIÓN}

En la primera fase, siendo esta de caracterización se le realiza una encuesta a los estudiantes, donde encontramos que la mayor parte de la población se encuentra en un estrato socioeconómico entre 1 y 2, además encontramos, que una parte de los estudiantes del curso posee una situación de vulnerabilidad ya que afirman que se pertenecen una fundación para jóvenes con problemáticas sociales. En este punto evidenciamos que se presenta una actitud de rebeldía frente a la clase, mostrando poco interés hacia la misma, como también se detecta un déficit en la línea de aprendizaje ya 


\title{
Bio - grafía. Escritos sobre la Biología y su Enseñanza. ISSN 2027-1034
}

\section{Edición Extraordinaria. p.p. 918 - 925}

\author{
Memorias del IX Encuentro Nacional de Experiencias en Enseñanza de la Biología y la \\ Educación Ambiental. IV Congreso Nacional de Investigación en Enseñanza de la \\ Biología.
}

que muchos de estos estudiantes dejan de estudiar por periodos de tiempo y no realizan un reforzamiento autónomo de los conceptos por su situación particular.

En cuanto a las ideas previas que poseen sobre el sistema digestivo dentro de la encuesta se realizó la siguiente pregunta: ¿Qué es el sistema digestivo y qué órganos lo componen? A la cual se presentan respuestas como:"es el aparato que digiere la comida llevándola al estómago" o "el sistema digestivo es todo lo que tenemos en el estómago y los órganos que lo componen son los pulmones, riñones, intestino grueso y intestino delgado". Lo que podemos evidenciar, es que, similar que lo indica Cubero (1998), en su investigación "Aprendizaje de la digestión en la enseñanza primaria" el estómago es tomado por los estudiantes como el órgano principal y donde ocurre todo el proceso de digestión y en algunos casos como un órgano que abarca a los demás. Sin embargo, contario a lo que ella indica acerca de que: "otra parte importante es la relacionada con la expulsión de los desechos donde los estudiantes creen que la comida no sale nunca del cuerpo o que puede ser eliminada oralmente (vomitando)" (cubero, 1998), encontramos en este caso, que poseen una idea clara de la expulsión de los desechos por medio de la deposición, solo que no tienen una idea clara de la ruta que sigue este proceso.

A partir de lo anterior y continuando con la segunda fase de articulación e implementación, se genera una unidad didáctica (Fonseca, 2016), en la cual se presenta una serie de actividades ya mencionadas en la metodología. Estas se formulan y realizan siguiendo los pasos que recomienda Pozo (2004), para las actividades de enseñanza y evaluación del enfoque de investigación dirigida; se desarrolla una actividad inicial, en la cual, se efectúa la presentación del tema buscando despertar el interés de los alumnos por el concepto que se va a abordar. Esta resulta ser exitosa en la mayoría de los estudiantes, ya que encontramos una respuesta favorable por parte de ellos hacia las metodologías que se plantean, aunque se mantienen reticentes a la idea del trabajo en grupo.

Continuando con la línea de trabajo del concepto de sistema digestivo y nutrición se implementa dentro de esta primera actividad, una segunda parte la cual busca que los estudiantes generen sus propias hipótesis a partir de un problema cotidiano; "¿cuáles son las principales macromoléculas en la digestión?, ¿qué sucede si comemos alimentos contaminados o con las manos sucias?, a lo cual encontramos respuestas como: " nos enfermamos pues hay virus y bacterias en nuestras manos y los carbohidratos y proteínas están expuestas a estos y no los digerimos " y " no pasa nada porque la comida está limpia y digerimos las proteínas y los carbohidratos que nos dan energía" (tomado textual).

Con esto evidenciamos como se presenta en primera medida, que los estudiantes luego de la introducción al tema han mostrado avance frente a los conceptos y tienen la capacidad de relacionar sus ideas previas con lo aprendido, ahora bien se les presenta un video el cual será la base para la resolución del problema y la confrontación de su hipótesis, con lo cual ellos lograr afirmar o corregir de ser necesario los errores que se presentaron en la formulación de su teoría. Es pertinente aclarar que para la realización de esta actividad y a partir de este punto se implementa la formación de grupos de trabajo, con el fin de fortalecer la colaboración y trabajo conjunto, a lo cual estos 


\title{
Bio - grafía. Escritos sobre la Biología y su Enseñanza. ISSN 2027-1034
}

Edición Extraordinaria. p.p. 918 - 925

\author{
Memorias del IX Encuentro Nacional de Experiencias en Enseñanza de la Biología y la \\ Educación Ambiental. IV Congreso Nacional de Investigación en Enseñanza de la \\ Biología.
}

responden en un principio de forma reacia, ya que solo querían trabajar con los amigos más cércanos dentro del curso o de lo contrario de manera individual.

La segunda y tercera actividad se presentan por medio de una UVE HEURISTICA, en la cual se plantea un problema, se formula una hipótesis y el análisis de resultados, es evidente que al igual que lo menciona Pozo (2004), se ponen en marcha por parte de los estudiantes una serie de estrategias y planifican de la forma más detallada, el modo en que van a dar solución al problema. Observamos que a medida que se desarrollan las clases los estudiantes presentan un cambio en la disposición frente a la metodología de trabajo grupal ya que dan cuenta de que es más fácil poner en marcha sus estrategias de trabajo y les facilita el análisis de los resultados, así como lo afirma Morales, et al. (2013), el "Saber hacer" "requiere por lo general realizar una secuencia de pasos, o secuencia de acciones para lo cual se requiere la adquisición de las habilidades y destrezas necesarias, los elementos que intervienen y cómo trabajarlos"(p.5).Estos adquieren un método particular y que más se les facilita para la complementación y culminación de las tareas dispuestas por ellos mismos. Ahora, en el desarrollo de estas actividades se observa que los alumnos identifican las prácticas experimentales y la investigación dirigida como un procedimiento valioso para la resolución de problemas.

Al finalizar la implementación de las actividades citadas, dentro de la unidad didáctica decidimos realizar una última actividad complementaria que se baso en la generación de un foro participativo, iniciando con la pregunta ¿qué es mejor, comer todo tipo de alimentos o ser vegetariano? generando una discusión por parte de los estudiantes y regulada por nosotros. En la que se obtuvo conclusiones como: "es mejor comer todo tipo de alimentos ya que en la carne y el pollo encontramos las proteínas que nos dan energía a nuestro cuerpo" para contradecir encontramos la siguiente opinión: "también se puede ser vegetariano ya que las lentejas y garbanzos y otros alimentos también tienen proteínas y no se necesita la carne" con respuestas como estas, evidenciamos el progreso conceptual que se presento en los estudiantes y como estos fueron capaces de explicar las cosas con sus propias palabras, articulando sus ideas previas y los nuevos conceptos adquiridos, desde su entendimiento individual y grupal comprobando que la investigación dirigida, al situar al alumno en el contexto que vive un científico lo motiva a la movilización conceptual autónoma, y al no verse presionado por una calificación que busca un saber de todo o nada, es capaz de arriesgarse a plantear una solución de una hipótesis generada a partir de un problema sin una respuesta exacta. Generando en él un cambio conceptual, procedimental y actitudinal frente a los nuevos conocimientos, adicional se evidencia como se formo una memoria final en la cual los alumnos son capaces, no solo de examinar los resultados obtenidos frente a un problema planteado, sino que también, de analizar y elegir sus propias estrategias de resolución; siendo la investigación dirigida un enfoque eficaz para la enseñanza del sistema digestivo y nutrición. 
Bio - grafía. Escritos sobre la Biología y su Enseñanza. ISSN 2027-1034

Edición Extraordinaria. p.p. 918 - 925

Memorias del IX Encuentro Nacional de Experiencias en Enseñanza de la Biología y la Educación Ambiental. IV Congreso Nacional de Investigación en Enseñanza de la Biología.

\section{CONCLUSIONES}

La investigación dirigida como modelo para la enseñanza del sistema digestivo resulta ser muy productiva ya que brinda herramientas necesarias para generar una articulación de sus ideas previas con los conceptos adquiridos y movilizar el interés de los estudiantes al involucrarlos en el contexto similar al que vive un científico.

La adquisición de conceptos es asimilada de manera rápida cuando se motiva al estudiante a desarrollar una hipótesis desde su cotidianidad y por medio de una investigación dirigida siendo continua, no generando presión por una calificación cuantitativa.

El trabajo en grupo y la actitud participante, fortalece la adquisición de procedimientos que facilitan la resolución de problemas y a su vez mejora la disposición y el respeto frente a su entorno generando una motivación de manera individual y colectiva en el trabajo investigativo.

\section{BIBLIOGRAFÍA}

Banet, E. y Núñez, F. (1992). Enseñanza de las ciencias, 10 (2), pág. 139-147. La digestión de los alimentos: un plan de actuación en el aula fundamentado en una secuencia constructivista del aprendizaje.

Fonseca, 2016. Guía de trabajo: Diseño unidad didáctica. Programa: Aula experimental. Febrero a junio de 2016.

Gil - Pérez, D. (1993). Contribución de la Historia y Filosofía de las ciencias al desarrollo de un modelo de enseñanza/aprendizaje como investigación,Enseñanza de las Ciencias, 11(2), 197-212.

Morales Morgado, Erla M.; García Peñalvo, Francisco; Campos Ortuño, Rosalynn A.; Astroza Hidalgo, Carlos; "Desarrollo de competencias a través de objetos de aprendizaje" RED. Revista de Educación a Distancia, núm. 36, marzo, 2013, pp. 1-19 Universidad de Murcia, España.

Pérez y Cubero, R. (1998). Aprendizaje de la digestión en la enseñanza primaria [Versión electrónica]. Revista Alambique 16.

Pozo Juan Ignacio, Gómez Crespo Miguel. Aprender y enseñar ciencia del conocimiento cotidiano al científico, ediciones morales s.l. Madrid .2004

Sánchez Ana. (2013). enseñanza - aprendizaje del concepto de digestión humana en estudiantes del grado sexto. 